\title{
Visual servo control for the underwater robot station-keeping
}

\author{
Chao-Lin Kuo \\ Department of Maritime Information and \\ Technology \\ National Kaohsiung Marine University \\ Kaohsiung, Taiwan \\ clkuo@webmail.nkmu.edu.tw \\ Chia-Hung Lin \\ Department of Electrical Engineering \\ Kao Yuan University \\ Kaohsiung, Taiwan \\ t21025@cc.kyu.edu.tw
}

\author{
Long-Yi Chang ${ }^{1}$, Ying-Che Kuo ${ }^{2}$ \\ Department of Electrical Engineering \\ National Chin-Yi University of Technology \\ Taichung, Taiwan \\ 1lychang@ncut.edu.tw \\ ${ }^{2}$ kuoyc@ncut.edu.tw \\ Kuei-Mei Lin \\ Department of Maritime Information and \\ Technology \\ National Kaohsiung Marine University \\ Kaohsiung, Taiwa \\ gracelin@mail.ssvs.tn.edu.tw
}

\begin{abstract}
This paper presents a visual servo and fuzzyPID controller to enable underwater robot stationkeeping. Movements of underwater robots are easily disturbed by water flow or external factors in a deepwater environment. In this study, control systems are used to adjust the underwater robot's propeller, which reduces chattering of the robot's postures, and visual target feedback and an accelerometer are employed to control its station-keeping. Results of a station-keeping test confirm the applicability and effectiveness of the proposed controller.
\end{abstract}

Keywords- Station-keeping; fuzzy-PID controller; visual servo control

\section{INTRODUCTION}

Underwater robot refers to the cabin is not equipped with underwater vehicles, many underwater research and exploration also need to use the underwater robot to complete, such as accident ship salvage, the sea Pipeline exploration, geological exploration of the waters and reservoir blockage investigation and so on. In recent years, several studies have been conducted to improve underwater robots, and these have focused on improving the associated control technology, institutional design, machine vision, and have involved the application of artificial intelligence [1-8]. However, high-tech research required is challenging and complex, and involves consideration of integrated mechanisms, automatic control, ship electromechanical integration, embedded system control, real-time digital signal processing, image processing and recognition, artificial intelligence, and expert systems. In addition, such research involves the application of self-learning theory, power management, control theory, and applied technology, and it also requires knowledge in the field of human and physical development. Traditionally, the differential or integral of the integer order has often been used in physical phenomenon or for the control of a system. For example, speed can be obtained through displacing the displacement, the curve can be integrated to obtain the area, and fuzzy PD or PID controllers are often applied in the control system, thereby providing good effects $[3,6,8,9]$.

Sjored and José proposed tracking and control aspects for automatic vision station-keeping with underwater remotely operated vehicles [2]. Incorporation of sliding mode control and a first-order low-pass filter with a new adaptive PID controller was proposed for tracking the control task of an uncertain nonlinear system [3]. In addition, Fossen et al. suggested a simple searching technique for obstacleavoidance of autonomous underwater vehicles in varying ocean environments, by using the self-tuning fuzzy controller [7]. Furthermore, Kuo et al. employed an embedded system to design the PID controller for underwater robot station-keeping.

The aim of this paper is to design a vision servo and fuzzy-PID controller to enable underwater robot station-keeping. The vision system is employed with respect to target and distance measurements, and the fuzzy-PID controller is used for station-keeping of the underwater robot. The remainder of this paper is organized as follows. Section 2 describes the hardware architecture of the underwater robot, Section 3 presents the fuzzy-PID controller used in the underwater robot's design, Section 4 presents results of an experimental test that confirm the applicability and effectiveness of the proposed control law, and conclusions are given in Section 5.

\section{THE UNDERWATER ROBOT}

Development of the underwater robot is described in this section (and is shown in Fig. 1). Figure 2 shows the electronic architecture of the underwater robot, which is fitted with a PC and embedded system (NI myRIO): the PC is located on the surface and the embedded system is contained in the underwater robot. The PC communicates with the embedded system by Ethernet protocol and a wireless router, and the system HMI (Human Machine Interface) is developed within 
the surface PC. Figure 3 shows the developed HMI, which displays a camera image, depth of the underwater robot, vertical thruster control voltage, three-axis inclination, and the real-time position of underwater robot. Tow power is supplied to the thrusters and the control system of the robot. The embedded system's functions are connected to the PC, the control thruster, the visible image, and for reading and processing the sensor signal (via a depth sensor, gyroscope, and accelerometer).

Figure 1. Underwater robot prototype.

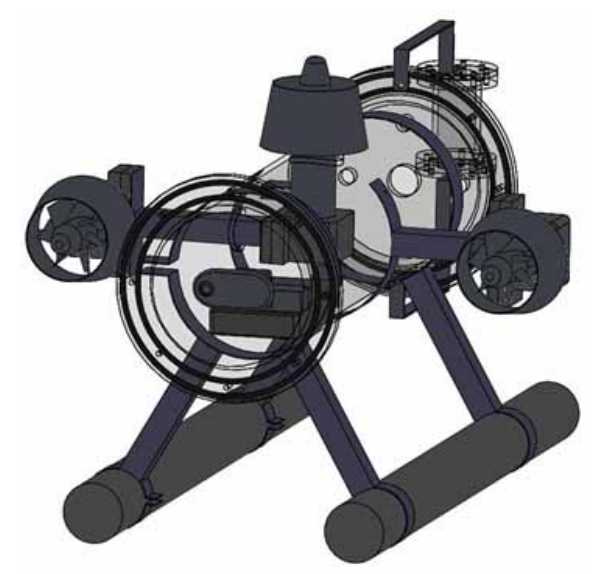

Figure 2. Electronic architecure.

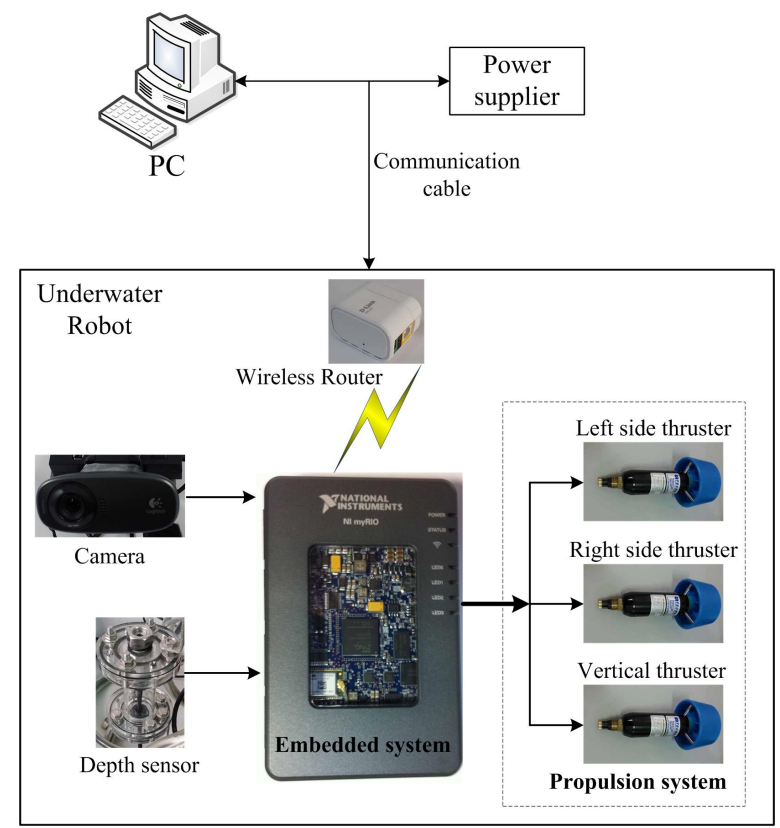

A webcam (Logitech HD Webcam C310, resolution $1280 \times 720 \mathrm{p}$ ) is used to capture images and is connected to the embedded system via a USB. Thrusters (models 260 and 300 from Tecnadyne, Inc.) are used to control the position and motion of the robot: model 300 thruster is used to control its depth and model 260 is used to control movement and direction. Underwater states, such as the robot's environment and depth, are provided by the sensing system, and information pertaining to this is displayed on the system HMI and control system. A pressure transducer (P51 from SSI Technologies, Inc.) is used to measure the robot's depth [8], and the three-axis inclination of the robot can be detected using a gyroscope and accelerometer via myRIO. The estimated water depth, $h$ can be described as

$$
h\left(v_{h}\right)=2.599 v_{h}-1.520
$$

where $h\left(v_{h}\right)$ is the estimated depth, and $v_{h}$ is the measurement voltage of the pressure transducer circuit.

Figure 3. HMI of underwater robot.

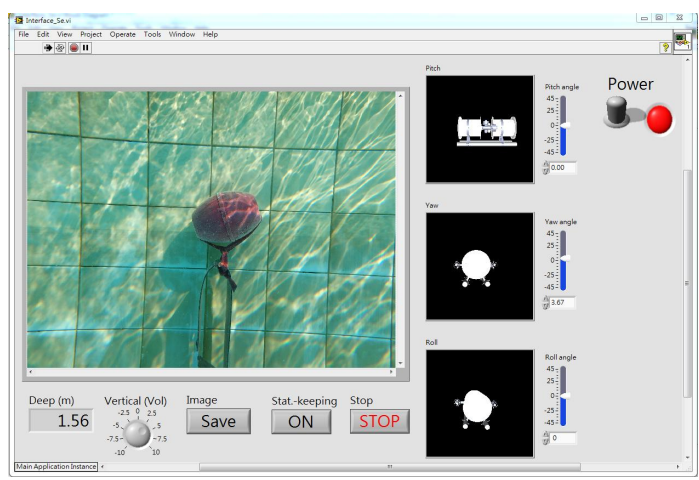

Figure 4. Earth and body-fixed coordinate of the underwater robot.<smiles>[Y]O[V]</smiles>

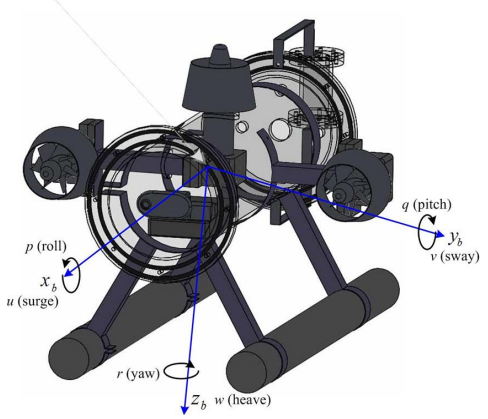

The Earth-fixed frame O-XYZ and body-fixed frame o-xyz are shown in Fig. 4; these coordinate systems are used to analyze the robot's motions. The underwater robot model can be given as follows [10],

$$
M \dot{v}+C(v) v+D(v) v+g(\eta)+d=\tau
$$

where $v$ is a velocity state vector, $\eta$ is a Euler parameter representing attitude, $M$ is the inertia matrix (including hydrodynamic added mass), $C$ is the matrix of Coriolis and centripetal terms (including hydrodynamic Coriolis and centripetal added mass), $D$ is the hydrodynamic damping matrix, $g$ is gravity, 
buoyancy force, and moment vector, and $\tau$ is a vector of the control inputs.

\section{FUZZY-PID CONTROLLER DESIGN}

This section presents the fuzzy-PID controller design for the underwater robot. In consideration of the robot's system (2), the target position is described as a state variable $P=\left[\begin{array}{lll}x & y & z\end{array}\right]^{T}$ in image detection and with the depth sensor. If the desired stationkeeping target state variable is defined as $P_{t}=\left[\begin{array}{lll}x_{t} & y_{t} & z_{t}\end{array}\right]^{T}$, then the target position error vector is defined as

$$
E=P_{t}-P=\left[\begin{array}{lll}
e_{x} & e_{y} & e_{z}
\end{array}\right]^{T} .
$$

\section{A. PID Controller}

The PID tracking control law for the underwater robot is defined as

$$
\tau_{i, P I D}=k_{p} e_{i}+k_{d} \frac{d e_{i}}{d t}+k_{i} \int e_{i} d t
$$

where $i$ is $x, y, z$, and $k_{p}, k_{d}$, and $k_{i}$ are positive constants

\section{B. Fuzzy Controller}

The fuzzy tracking control law for the underwater robot is defined as

$$
\tau_{i, f u z z y}=k_{f} \cdot F_{f u z z y}\left(e_{i}, \dot{e}_{i}\right)
$$

where $k_{f}$ is a positive constant and $F_{f u z z y}(\cdot, \cdot)$ is the fuzzy relation function of $e_{i}$ and $\dot{e}_{i}$ (values of $e_{i}$ and $\dot{e}_{i}$ are linguistic variables). The fuzzy rule can be used to design a sliding rules table [8], and fuzzy tracking control $\tau_{i, f u z z y}$ is calculated by the weighted average de-fuzzification method. In the embedded system, a look-up table scheme is used to implement the fuzzy rules.

Figure 5. Block diagram of the fuzzy-PID controller.

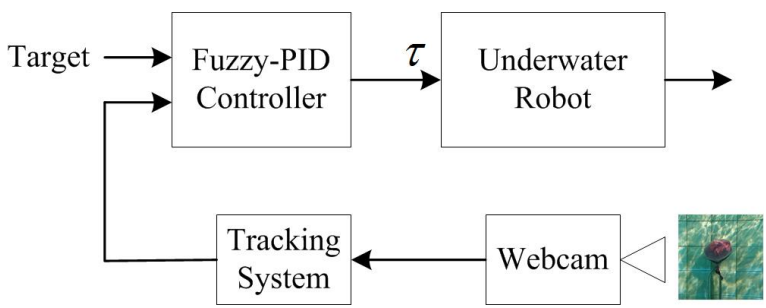

A fuzzy-PID controller is proposed for the underwater robot, in which both PID control (4) and fuzzy control (5) are used. The control law is as follows,

$$
\tau_{i}=\alpha_{i} \cdot \tau_{i, P I D}+\left(1-\alpha_{i}\right) \tau_{i, f u z z y}
$$

where $\alpha_{i}$ is an $\mathrm{S}$ function define as

$$
\alpha_{i}= \begin{cases}2\left(\frac{e_{i}}{b}\right)^{2} & ,\left|e_{i}\right|<\frac{b}{2} \\ 1-2\left(\frac{\left|e_{i}\right|-b}{b}\right)^{2} & , \frac{b}{2} \leq\left|e_{i}\right|<b \\ 1 & ,\left|e_{i}\right| \geq b\end{cases}
$$

where $b$ is positive constant. The proposed controller design for controlling station-keeping using the visual servo is shown in Fig. 5; a webcam is used to capture the image and the target position is determined and estimated by the algorithm in the tracking system.

\section{RESULTS AND DISCUSSION}

To determine the efficiency of the proposed control scheme, the underwater robot is tested in a swimming pool (Fig. 6 shows a photo of the experiment). The visual system can process 10 images per second. The test involves moving the underwater robot to a suitable depth, activating the lock target, and station-keeping. The target image is shown in Fig. 7 , and can be viewed from the HMI. The user sets the "vertical" knob to change the z-axis position of the robot from the HMI. When the robot approaches a target position, the webcam films an image of the target, and the user pushes the "Stat.-keeping" button. The proposed controller (6) is then applied to control the underwater robot, which enables control of station-keeping and locking-on to a target. Figure 8 shows a time response of the $\mathrm{z}$-axis for the underwater robot. Experimental results illustrate that the proposed control scheme is successful and that the robot effectively managed station-keeping and locking-on to a target. Therefore, the proposed scheme successfully realized visual servo control of the underwater robot.

\section{CONCLUSION}

In this paper, use of fuzzy-PID control with a visual servo was studied to enable station-keeping of an underwater robot. Results show that the webcam tracking system detected the target and its central position. A fuzzy-PID hybrid-controller is introduced and employed to control the states of the underwater robot. Experimental results conducted in a swimming pool demonstrate that the robot effectively manages station-keeping and locking-on to a target using the proposed control scheme.

\section{ACKNOWLEDGMENT}

This work has been supported by the Ministry of Science Technology, R.O.C., under contract MOST 105-2221-E-022-011. 
Figure 6. The experiment photo.

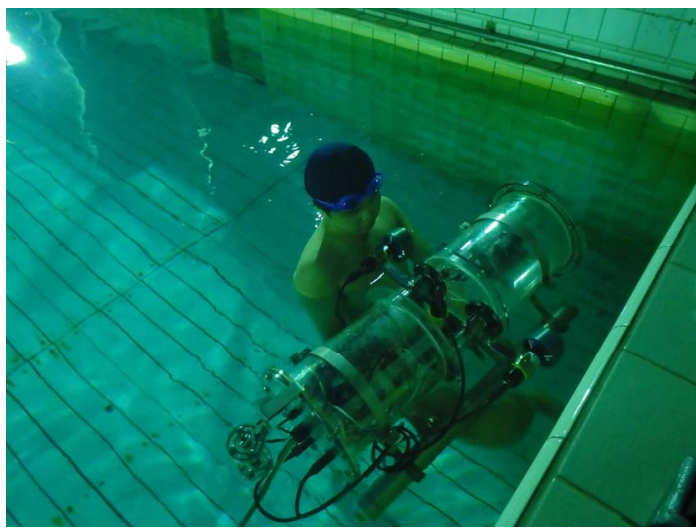

Figure 7. Target image.

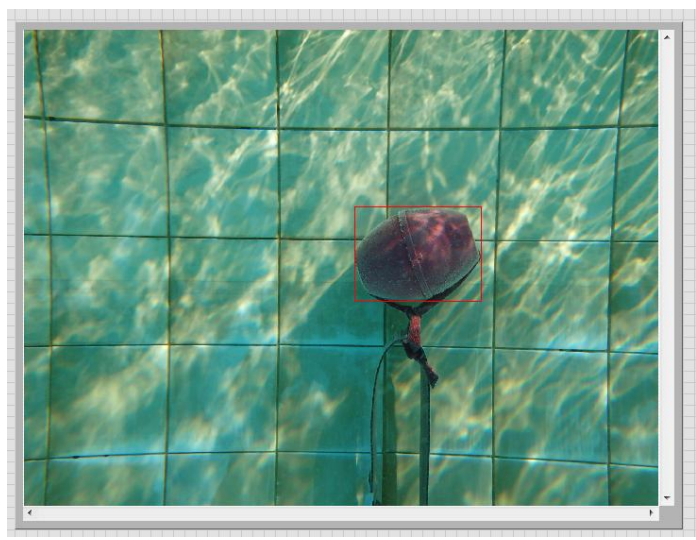

Figure 8 . The time response of z-axis.

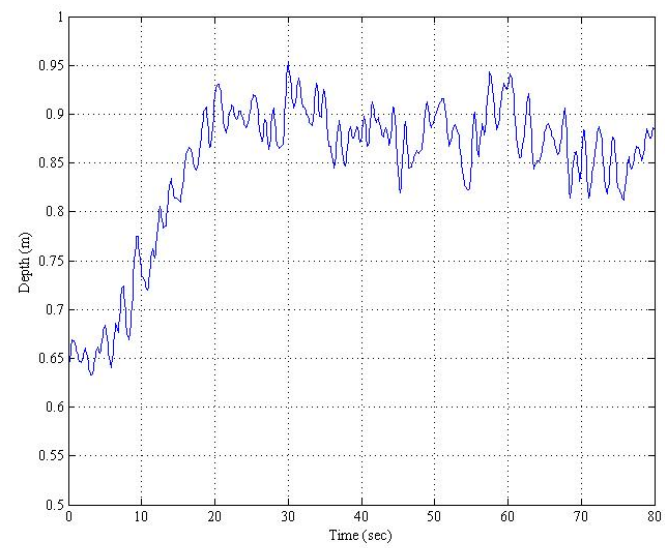

\section{REFERENCES}

[1] C. Silpa-Anan, T. Brinsmead, and S. Abdallah, "Preliminary experiments in visual servo control for autonomous underwater vehicle," IEEE/RSJ international conference on intelligent robotics and systems (IROS); 2001. Available from: http://www.syseng.anu.edu.au/rsl/.

[2] S. van der Zwaan and J. Santos-Victor, "Real-time visionbased station keeping for underwater robots," OCEANS, 2001. MTS/IEEE conference and exhibition, pp. vol. 2, pp. 10581065,2001

[3] N. Q. Hoang and E. Kreuzer, "Adaptive PD-controller for positioning of a remotely operated vehicle close to an underwater structure: Theory and experiments," Control Engineering Practice, No. 15, pp. 411-419, 2007.

[4] G.-V. Luis Govinda, S.-J. Tomás, B.-S. Manuel, N.-B. Luciano, H.-A. Rodrigo and C.-L. José Antonio, "Modelling, Design and Robust Control of a Remotely Operated Underwater Vehicle," International Journal of Advanced Robotic Systems, vol. 11, no. 1, pp. 1-16, 2014.

[5] C. Barbălată, V. D. Carolis, M. W. Dunnigan, Y. Pétillot and D. Lane, "An adaptive controller for autonomous underwater vehicle," in Proceedings of 2015 IEEE/RSJ International Conference on Intelligent Robots and Systems, pp. 16581663, 28 Sept - 2 Oct, 2015.

[6] T. Chamsai, P. Jirawattana and T. Radpukdee, "Robust adaptive PID controller for a class of uncertain nonlinear systems: an application for speed tracking control of an SI engine," Mathematical Problems in Engineering, vol. 2015, pp. 1-13, 2015.

[7] M.-C. Fang, S.-M. Wang, M.-C. Wu and Y.-H. Lin, "Applying the self-tuning fuzzy control with the image detection technique on the obstacle-avoidance for autonomous underwater vehicles," Ocean Engineering, vol. 93, pp. 11-24, 2015.

[8] C.-L. Kuo, C.-K. Tsui, N.-S. Pai, C.-H. Lin, S.-C. Chen and P.-W. Li, "A PID controller for the underwater robot stationkeeping," INDIN 2016 IEEE International Conference on Industrial Informatics, pp. 1242-1246, July 18-21, 2016

[9] J.-L. Chen, C.-L. Kuo, S.-J. Chen, C.-C. Kao, T.-S. Zhan, C.H. Lin, and Y.-S. Chen, "DC-side fault detection for photovoltaic energy conversion system using fractional-order dynamic-error-based fuzzy petri net integrated with intelligent meters," IET Renewable Power Generation,Vol. 10, No. 9, pp. 1318-1327, 2016.

[10] T. I. Fossen, Guidance and control of ocean vehicles, University of Trondheim Norway: Wiley, 1994. 